\title{
Âgé et sexy : un sujet tabou ?
}

\author{
Nora Tschanz et Céline Uehlinger
}

La sexualité est la chose la plus normale au monde et est omniprésente aujourd'hui dans tous les médias. Pourtant la relation entre sexualité et vieillesse relève du défi. Qui s'est déjà entretenu avec ses (grands-) parents au sujet de leur vie sexuelle ? Dans leur pratique de soins, les auteures ont vécu la question de la sexualité au 3e âge comme tabou et ont décidé de faire de la sexualité dans les institutions de soins aux personnes âgées le sujet de leur travail de Bachelor.

\section{Des besoins même pendant la vieillesse}

« J'aimerais baiser de nouveau ! », c'est ainsi que s'est exprimé un résident d'une institution de soins aux personnes âgées. Ce genre de déclarations signifie que même les personnes âgées ont des besoins sexuels. Le terme « sexualité des personnes âgées » correspond à des besoins et à leur expression pendant la vieillesse. Ni l'âge avancé ni l'entrée dans une institution pour personnes âgées ne signifie qu'une personne est sexuellement inactive. Même une étude montre qu'en Suisse 22,2\% des femmes et 23,3\% des hommes de plus de 75 ans exprimaient plusieurs fois par semaine un intérêt pour le sexe (Bucher et al., 2003). La sexualité est comprise comme un aspect important de la vie et pertinent pendant toute la durée de la vie. Elle peut être vécue et exprimée par des pensées, des fantasmes, du désir, des représentations, des attitudes, des valeurs, le comportement, la pratique, des rôles ou des relations.

\section{Questions et réponses}

Dans le cadre de leur travail de Bachelor, les auteures ont creusé les questions suivantes : quelle attitude les employés des institutions de soins aux personnes âgées ont-ils envers la sexualité de leurs résidents ou des personnes âgées ? Quels sont les facteurs qui influencent cette attitude ? Quelles actions et réactions des employés ont résulté de cette attitude par rapport à la sexualité des résidents ? Pour collecter des données, une étude de la littérature a été menée dans différentes bases de données. Avec des mots clés correspondants, trois études quantitatives, deux études qualitatives et trois « méthodes mixtes », c'est-à-dire la mise en parallèle des méthodes quantitatives et qualitatives, ainsi qu'une revue ont été trouvées.

Le résumé des résultats a montré que l'attitude des employés envers la sexualité des personnes âgées n'était ni clairement restrictive ni clairement tolérante. Leur attitude est influencée entre autres par des facteurs sociodémographiques. Ainsi plus les employés sont âgés et ont une position politique libérale, plus leur niveau de formation est élevé et plus leur expérience professionnelle est grande, plus ils sont tolérants. La fréquence de participation des employés aux formations sur le sujet de la sexualité chez 
les personnes âgées a un effet positif sur leur attitude. La position de base de l'institution et de ses directives est également déterminante. La réaction des employés sur la sexualité des personnes âgées est par conséquent très différente. L'attirance envers une deuxième personne crée par exemple comme réactions le sentiment de dégoût, de gêne et de malaise ainsi que l'ignorance voire l'interdiction de la sexualité.

\section{Possibilité d'influence}

Comme la position de base d'une institution influence la pratique des soignants, il est important d'avoir des directives représentatives de l'éthique qui font de la sexualité un thème de discussion. Comme les formations continues sur le thème de la sexualité des personnes âgées entraînent une attitude plus tolérante, les programmes de formation peuvent apporter d'importantes contributions au thème de la sexualité des personnes âgées et à l'intégration du thème dans le programme de formation aux soins. Afin que les personnes âgées puissent également vivre leur sexualité en institution sans que cela ne dérange les autres, elles ont besoin d'intimité. La protection de l'intimité peut être améliorée par des interventions faciles à mettre en place :

- Frapper à la porte

- Panonceau «Ne pas déranger»

- Définir dans l'emploi du temps quotidien des moments où il ne faut pas déranger les résidents

- Proposer des chambres simples ou doubles suivant les besoins du résident, etc.

\section{Sexualité des personnes âgées et technologie ?}

Qu'est-ce que l'un peut bien avoir avec l'autre ? Non, il ne s'agit pas de technologies spéciales en matière de sexe, mais bien plus de la question de savoir dans quelle mesure les accesoires technologiques peuvent aider directement ou indirectement l'expérience et l'expression de la sexualité pendant la vieillesse en institution de soins également. Ainsi, une " pièce pour câlins » peut par exemple être aménagée avec de la musique choisie individuellement et des jeux de lumière variables et donc créer une ambiance intime qui suscite le désir de se rapprocher et permet d'être ensemble sans être dérangé. La " pièce pour câlins » peut également être équipée de sièges massants, de coussins chauffants et de « sextoys » qui favorisent l'expérience sexuelle. Dans tous les cas, des stimulations qui valent la peine d'y penser.

Les auteures se prononcent clairement pour le fait que la thématisation des besoins sexuels chez les personnes âgées représente à elle seule une étape importante de la détabouïsation. Leur travail de Bachelor devrait apporter sa contribution à ce sujet. 


\section{Littérature :}

Tschanz, N \& Uehlinger, C. (2015) Alt sexy - Thema oder Tabu? Eine Analyse der Haltung und der daraus folgenden Reaktionen der Mitarbeitenden von Alterspflegeinstitutionen bezüglich Alterssexualität. Bachelorarbeit „Pflege“, Departement Gesundheit der Züricher Hochschule für angewandte Wissenschaften (zhaw).

Une littérature plus complète peut être obtenue auprès des auteures.

Auteures : Nora Tschanz et Céline Uehlinger, BSc en soins infirmiers.

Contact : tschanora@hotmail.com; celineuehlinger@hotmail.com 